\title{
IDENTITIES INVOLVING THE COEFFICIENTS OF A CLASS OF DIRICHLET SERIES. V
}

\author{
BY \\ BRUCE C. BERNDT( $\left.{ }^{1}\right)$
}

\begin{abstract}
We derive various forms of the Voronoï summation formula for a large class of arithmetical functions. These arithmetical functions are generated by Dirichlet series satisfying a functional equation with certain gamma factors. Using our theorems, we establish several arithmetical identities.
\end{abstract}

1. Introduction. Let $f$ be of bounded variation on $[a, b]$. Then the Poisson summation formula

$$
\frac{1}{2} \sum_{n=a}^{b}\{f(n+0)+f(n-0)\}=\int_{a}^{b} f(x) d x+2 \sum_{n=1}^{\infty} \int_{a}^{b} f(x) \cos (2 \pi n x) d x
$$

is valid, where the ' indicates that when $n=a$ only the term $\frac{1}{2} f(a+0)$ is counted and when $n=b$ only the term $\frac{1}{2} f(b-0)$ is counted.

In 1904, Voronoï [30] made the following conjecture which can be regarded as a generalization of the Poisson formula. Let $a(n)$ be an arithmetical function and let $f$ be continuous on $(a, b)$ with only a finite number of maxima and minima on $(a, b)$. Then there exist analytic functions $\delta(x)$ and $\alpha(x)$, depending only on $a(n)$ and not on $f(x)$, such that

$$
\sum_{a \leq n \leq b}^{\prime} a(n) f(n)=\int_{a}^{b} f(x) \delta(x) d x+\sum_{n=1}^{\infty} a(n) \int_{a}^{b} f(x) \alpha(n x) d x,
$$

where the ' has the same meaning as above. If $a(n) \equiv 1$, the conjecture is true, for by Poisson's formula we can take $\delta(x) \equiv 1$ and $\alpha(x)=2 \cos (2 \pi x)$. Voronoï was able to establish his conjecture for the divisor function $d(n)$ when $0<a<b<\infty$. In this case, $\delta(x)=\log x+2 \gamma$, where $\gamma$ denotes Euler's constant, and $\alpha(x)$ $=4 K_{0}\left(4 \pi x^{1 / 2}\right)-2 \pi Y_{0}\left(4 \pi x^{1 / 2}\right)$, where $K_{0}$ and $Y_{0}$ are the Bessel functions usually so denoted. In 1929, Koshliakov [23] gave a considerably shorter proof of Voronoï's formula, but with the assumption that $f$ be analytic. Dixon and Ferrar [9] in 1931 gave a still shorter proof with the hypothesis that $f \in C^{(2)}[a, b]$. In 1932, Wilton [34] proved the Voronoï formula under the assumption that $f$ be of bounded variation

Received by the editors July 14, 1970.

AMS 1969 subject classifications. Primary 1043; Secondary 1041.

Key words and phrases. Arithmetical function, Voronoï summation formula, arithmetical identities, functional equation with gamma factors.

(1) Research partially supported by NSF grant GP-21335. 
on $[a, b]$. He also extended the formula to include the cases $a=0$ and $b=\infty$. A further refinement for the case $a=0$ was later made by Dixon and Ferrar [12].

The case $a(n)=r_{2}(n)$, the number of representations of $n$ as the sum of two squares, has also been given considerable attention. Voronoï [31] was also the first to give a formula in this case. Here $\delta(x) \equiv \pi$ and $\alpha(x)=\pi J_{0}\left(2 \pi x^{1 / 2}\right)$, where $J_{0}$ denotes the usual Bessel function. Voronoï's formula was also given by Sierpiński [28]. Landau [24] has established the formula when $f$ is of bounded variation on $[a, b]$, $0 \leqq a<b<\infty$. The extension to $b=\infty$ was made by Dixon and Ferrar [10].

The Voronoï conjecture has been established for several other arithmetical functions. In the derivation of these formulae, one usually first establishes an identity for $\sum_{n \leqq x} a(n)$ in terms of an infinite series of Bessel functions. A second observation is that, for every Voronoï summation formula that has been established, the arithmetical function $a(n)$ is generated by a Dirichlet series satisfying a functional equation with gamma factors. Our objective is to develop general Voronoï summation formulae for arithmetical functions satisfying the aforementioned two properties.

A survey of all of the established results indicates that the hypotheses under which the Voronoï formula has been proved generally fall in three classes. First, $f$ is "smooth", i.e., $f \in C^{(1)}[a, b]$ or $C^{(2)}[a, b]$. In $\S 3$ we prove general theorems for such functions. Secondly, $f$ is of bounded variation on $[a, b]$. In $\$ 4$ we establish the Voronoï formula for such $f$. Our results here are not as complete as for the first class because of the difficulty caused by $a=0$.

Lastly, a third approach uses the theory of functions in $L^{2}(0, \infty)$ and the theory of Mellin or Fourier or Hankel transforms. Such an approach was inaugurated by Ferrar [13], [14] and Guinand [16], [17], [19]. With this approach a formula for $r_{2}(n)$ is proved by Pearson [27] and one for $d(n)$ by Nasim [26]. We do not attempt to give a general theory using this approach. In fact, the aforementioned papers of Ferrar and Guinand give general theorems.

Although general theorems have been established using transform techniques, only a couple of attempts have been made to obtain general results for "smooth" functions or for functions of bounded variation. The first general theorem was given by Koshliakov [22] who assumed that $\{a(n)\}$ is generated by an exponential series satisfying a "theta-relation", and that $f$ is analytic. A second general theorem is given by us in [2] where it is assumed that the generating Dirichlet series satisfies a functional equation with a simple gamma factor, i.e., $\Gamma(s)$, and that the series has an analytic continuation which is entire or has at worst only one simple pole, that at a certain specified point.

In the sequel we let $s$ be complex with $\sigma=\operatorname{Re}(s)$, and write $\sum$ for $\sum_{n=1}^{\infty} . A$ always denotes a positive constant, not necessarily the same with each occurrence.

2. The arithmetical functions. Let $\left\{\lambda_{n}\right\}$ and $\left\{\mu_{n}\right\}$ be two sequences of positive numbers strictly increasing to $\infty$, and $\{a(n)\}$ and $\{b(n)\}$ two sequences of complex 
numbers not identically zero. Suppose that

$$
\varphi(s)=\sum a(n) \lambda_{n}^{-s} \text { and } \psi(s)=\sum b(n) \mu_{n}^{-s}
$$

each converge in some half-plane and have abscissae of absolute convergence $\sigma_{a}$ and $\sigma_{a}^{*}$, respectively. Let $\Delta(s)$ denote one of the following three gamma factors: $\Gamma(s), \Gamma\left(\frac{1}{2} s\right) \Gamma\left(\frac{1}{2}\{s-p\}\right)$, or $\Gamma^{2}\left(\frac{1}{2}\{s+1\}\right)$, where $p$ is an integer. Let $r$ be, in the three cases respectively, arbitrarily real, $p+1$, and 1 . Then we say that $\varphi$ and $\psi$ satisfy the functional equation $\Delta(s) \varphi(s)=\Delta(r-s) \psi(r-s)$ if there exists a meromorphic function $\chi$ with the following properties:

(i) $\chi(s)=\Delta(s) \varphi(s), \sigma>\sigma_{a}, \chi(s)=\Delta(r-s) \psi(r-s), \sigma<r-\sigma_{a}^{*}$;

(ii) $\lim _{|\operatorname{Im}(s)| \rightarrow \infty} \chi(s)=0$, uniformly in every interval $-\infty<\sigma_{1} \leqq \sigma \leqq \sigma_{2}<\infty$;

(iii) the poles of $\chi$ are confined to some compact set.

For such arithmetical functions Chandrasekharan and Narasimhan [6], [7] have established fundamental identities involving Bessel functions. See also [3] and [5]. Let $q$ be a nonnegative integer and $x>0$. Define

$$
A_{q}(x)=\frac{1}{\Gamma(q+1)} \sum_{\lambda_{n} \leq x}^{\prime} a(n)\left(x-\lambda_{n}\right)^{q},
$$

where the ' indicates that if $q=0$ and $\lambda_{n}=x, a(n)$ is to be multiplied by $\frac{1}{2}$. Define also

$$
Q_{q}(x)=\frac{1}{2 \pi i} \int_{C_{q}} \frac{\Gamma(s) \varphi(s)}{\Gamma(s+q+1)} x^{s+q} d s,
$$

where $C_{q}$ is a cycle enclosing all of the integrand's poles. Furthermore, define

$$
D_{q}(x)=A_{q}(x)-Q_{q}(x) .
$$

Suppose that for $\sigma>\sigma_{a}^{*}$,

$$
\sup _{0 \leqq n \leqq 1}\left|\sum_{m^{2} \leqq \mu_{n} \leqq(m+h)^{2}} b(n) \mu_{n}^{1 / 2-\sigma}\right|=o(1)
$$

as $m$ tends to $\infty$. Then, for $q>2 \sigma_{a}^{*}-r-3 / 2$,

$$
D_{q}(x)=\sum \frac{b(n)}{\mu_{n}^{r+q}} I_{q}\left(\mu_{n} x\right),
$$

where in the three cases $\Delta(s)=\Gamma(s), \Gamma\left(\frac{1}{2} s\right) \Gamma\left(\frac{1}{2}\{s-p\}\right)$, and $\Gamma^{2}\left(\frac{1}{2}\{s+1\}\right)$ we have, respectively,

$$
\begin{aligned}
I_{q}(x)= & x^{(r+q) / 2} J_{r+q}\left(2 x^{1 / 2}\right), \\
= & x^{(p+q+1) / 2} 2^{-q}\left\{\cos \left(\frac{1}{2}\{p+1\} \pi\right) J_{p+q+1}\left(4 x^{1 / 2}\right)\right. \\
& \left.\quad-\sin \left(\frac{1}{2}\{p+1\} \pi\right)\left[Y_{p+q+1}\left(4 x^{1 / 2}\right)+\left(2(-1)^{p+q} / \pi\right) K_{p+q+1}\left(4 x^{1 / 2}\right)\right]\right\}, \\
= & x^{(q+1) / 2} 2^{-q}\left\{Y_{q+1}\left(4 x^{1 / 2}\right)+\left(2(-1)^{q+1} / \pi\right) K_{q+1}\left(4 x^{1 / 2}\right)\right\} .
\end{aligned}
$$


The series of Bessel functions in (2.2) converges uniformly on any interval in $x>0$ where $A_{q}(x)-Q_{q}(x)$ is continuous. The series converges boundedly on any compact interval in $(0, \infty)$ if $q=0$.

Note that from standard differentiation formulae for Bessel functions we have

$$
I_{q}^{\prime}(x)=I_{q-1}(x) \text {. }
$$

In the sequel we shall assume that (2.2) is valid for $q=0$.

3. A Voronoi summation formula for "smooth" functions. The proofs of the theorems in this section are very easy.

THEOREM 1. Let $f \in C^{(1)}(0, \infty)$. Then, if $0<a<\lambda_{1}<x<\infty$,

$$
\sum_{\lambda_{n} \leqq x}^{\prime} a(n) f\left(\lambda_{n}\right)=\int_{a}^{x} Q_{0}^{\prime}(t) f(t) d t+\sum \frac{b(n)}{\mu_{n}^{r-1}} \int_{a}^{x} I_{-1}\left(\mu_{n} t\right) f(t) d t .
$$

Proof. By partial summation and (2.1),

$$
\begin{aligned}
\sum_{\lambda_{n} \leqq x}^{\prime} a(n) f\left(\lambda_{n}\right) & =A_{0}(x) f(x)-\int_{a}^{x} A_{0}(t) f^{\prime}(t) d t \\
& =\left\{Q_{0}(x)+D_{0}(x)\right\} f(x)-\int_{a}^{x}\left\{Q_{0}(t)+D_{0}(t)\right\} f^{\prime}(t) d t .
\end{aligned}
$$

Since $D_{0}(t)$ converges boundedly on $[a, x]$, we may invert the order of summation and integration $[29$, p. 41$]$ to obtain

$$
\int_{a}^{x}\left\{Q_{0}(t)+D_{0}(t)\right\} f^{\prime}(t) d t=\int_{a}^{x} Q_{0}(t) f^{\prime}(t) d t+\sum \frac{b(n)}{\mu_{n}^{r}} \int_{a}^{x} I_{0}\left(\mu_{n} t\right) f^{\prime}(t) d t .
$$

The integral involving $Q_{0}(t)$ clearly exists because of assumption (iii). Integrating by parts with the aid of (2.3), we obtain

$$
\sum_{\lambda_{n} \leqq x}^{\prime} a(n) f\left(\lambda_{n}\right)=\left\{Q_{0}(a)+D_{0}(a)\right\} f(a)+\int_{a}^{x} Q_{0}^{\prime}(t) f(t) d t+\sum \frac{b(n)}{\mu_{n}^{r-1}} \int_{a}^{x} I_{-1}\left(\mu_{n} t\right) f(t) d t .
$$

But $a<\lambda_{1}$, and so $Q_{0}(a)+D_{0}(a)=A_{0}(a)=0$. Thus, the proof is complete.

The inversion in order of summation and integration above is not necessarily justified if $a=0$. In Corollary 8 we shall state some conditions that insure the bounded convergence of $D_{0}(t)$ on $0 \leqq t \leqq x$ in the case $\Delta(s)=\Gamma(s)$. The following theorem is clear from the proof of Theorem 1.

TheOREM 2. Let $f \in C^{(1)}(0, \infty)$. Suppose that $D_{0}(0) f(0)<\infty$ and $\int_{0}^{x} Q_{0}^{\prime}(t) f(t) d t$ $<\infty$. Furthermore, assume that $D_{0}(t)$ converges boundedly on $0 \leqq t \leqq x$. Then (3.1) is valid with $a=0$.

We now prove two theorems which do not depend upon the bounded convergence of $D_{0}(t)$ on $0 \leqq t \leqq x$.

THEOREM 3. Let $f \in C^{(1)}[0, \infty)$. Suppose that the poles of $\varphi(s)$ are in the half-plane $\sigma>0$. Then (3.1) is valid with $a=0$. 
Proof. We shall show that letting $a$ tend to 0 in Theorem 1 is justified by continuity considerations.

Since the poles of $\varphi(s)$ lie in $\sigma>0$,

$$
\int_{0}^{x} Q_{0}^{\prime}(t) f(t) d t<\infty
$$

Thus, the first term on the right-hand side of (3.1) is continuous at $a=0$. Upon an integration by parts, the second term on the right-hand side of (3.1) becomes

$$
D_{0}(x) f(x)-D_{0}(a) f(a)-\int_{a}^{x} D_{0}(t) f^{\prime}(t) d t,
$$

where the inversion in order of summation and integration is justified as in the proof of Theorem 3.1. $A_{0}(a)$ is clearly continuous at $a=0$, and $Q_{0}(a)$ is continuous at $a=0$ by our assumption on the poles of $\varphi$. (3.2) is then continuous at $a=0$, and the proof is complete.

In some applications the above condition on $f$ at the origin is too strong. In the case $\Delta(s)=\Gamma(s)$ we are able to weaken this condition. Because $K_{v}$ and $Y_{v}$ have singularities at the origin, our proof is not valid for the other choices of $\Delta(s)$.

TheOREM 4. Let $f \in C^{(2)}(0, \infty)$. Suppose that $\Delta(s)=\Gamma(s)$ and that the poles of $\varphi$ lie in $\sigma>0$. Assume that $Q_{0}(t) f(t)$ and $Q_{1}(t) f^{\prime}(t)$ are continuous at $t=0$. Lastly, suppose that

Then,

$$
\int_{0}^{1} t^{r / 2+1 / 4}\left|f^{\prime \prime}(t)\right| d t<\infty
$$

$$
\begin{aligned}
& \sum_{\lambda_{n} \leqq x}^{\prime} a(n) f\left(\lambda_{n}\right) \\
& =\int_{0}^{x} Q_{0}^{\prime}(t) f(t) d t+\sum \frac{b(n)}{\mu_{n}^{(r-1) / 2}} \int_{0}^{x} t^{(r-1) / 2} J_{r-1}\left(2\left\{\mu_{n} t\right\}^{1 / 2}\right) f(t) d t .
\end{aligned}
$$

Proof. Proceed as in the proof of Theorem 3. From (3.2) and our hypotheses we need only to examine

$$
\sum \frac{b(n)}{\mu_{n}^{r}} \int_{a}^{x} I_{0}\left(\mu_{n} t\right) f^{\prime}(t) d t
$$

Upon an integration by parts, the above becomes

$$
D_{1}(x) f^{\prime}(x)-D_{1}(a) f^{\prime}(a)-\sum \frac{b(n)}{\mu_{n}^{r+1}} \int_{a}^{x} I_{1}\left(\mu_{n} t\right) f^{\prime \prime}(t) d t .
$$

Since $Q_{1}(a) f^{\prime}(a)$ is continuous at $a=0$, it suffices to show that

$$
\sum \frac{b(n)}{\mu_{n}^{(r+1) / 2}} \int_{0}^{1} t^{(r+1) / 2} J_{r+1}\left(2\left\{\mu_{n} t\right\}^{1 / 2}\right) f^{\prime \prime}(t) d t
$$

is absolutely convergent. For then the infinite series in (3.4) converges uniformly on $0 \leqq a \leqq 1$ and hence is a continuous function of $a$ on $[0,1]$. 
We divide the interval of integration in (3.5) into $\left[0,1 / \mu_{n}\right]$ and $\left[1 / \mu_{n}, 1\right]$. Since $J_{v}(s)=O\left(x^{v}\right)$ as $x$ tends to 0 , the integral over $\left[0,1 / \mu_{n}\right]$ is less than in modulus

$$
A \mu_{n}^{(r+1) / 2} \int_{0}^{1 / \mu_{n}} t^{r+1}\left|f^{\prime \prime}(t)\right| d t \leqq A \mu_{n}^{-1 / 4} \int_{0}^{1 / \mu_{n}} t^{r / 2+1 / 4}\left|f^{\prime \prime}(t)\right| d t .
$$

Since $J_{v}(x)=O\left(x^{-1 / 2}\right)$ as $x$ tends to $\infty$, the modulus of the integral over [1/ $\left.\mu_{n}, 1\right]$ is less than

$$
A \mu_{n}^{-1 / 4} \int_{1 / u_{n}}^{1} t^{r / 2+1 / 4}\left|f^{\prime \prime}(t)\right| d t .
$$

Now, since (2.2) is valid for $q=0$, we must have $0>2 \sigma_{a}^{*}-r-3 / 2$ or $r / 2+3 / 4>\sigma_{a}^{*}$. Hence, from (3.6) and (3.7) the modulus of (3.5) is less than

$$
A \sum \frac{|b(n)|}{\mu_{n}^{r / 2+3 / 4}} \int_{0}^{1} t^{r / 2+1 / 4}\left|f^{\prime \prime}(t)\right| d t<\infty
$$

and the proof of (3.3) is complete.

Of course, by subtraction a Voronoï formula may be given over any finite interval $[a, b], a \geqq 0$, from the above results. We now wish to replace $x$ by $\infty$ in these theorems.

TheOREM 5. Let $f \in C^{(2)}(0, \infty)$. Suppose that $D_{0}(x) f(x)$ and $x^{r / 2+1 / 4} f^{\prime}(x)$ tend to 0 as $x$ tends to $\infty$. Assume that, for $x>0$,

Then, if $0<a<\lambda_{1}$,

$$
\int_{x}^{\infty} t^{r / 2+1 / 4}\left|f^{\prime \prime}(t)\right| d t<\infty
$$

$$
\lim _{x \rightarrow \infty}\left(\sum_{\lambda_{n} \leqq x}^{\prime} a(n) f\left(\lambda_{n}\right)-\int_{a}^{x} Q_{0}^{\prime}(t) f(t) d t\right)=\sum \frac{b(n)}{\mu_{n}^{r-1}} \int_{a}^{\infty} I_{-1}\left(\mu_{n} t\right) f(t) d t,
$$

provided that the limit on the left side exists.

By assuming in addition either the hypotheses of Theorems 2, 3, or 4 we may let $a=0$ in Theorem 5. To determine if $D_{0}(x) f(x)$ tends to 0 as $x$ tends to $\infty$, we may consult a general theorem of Chandrasekharan and Narasimhan [7, Theorem 4.1].

Proof. It is sufficient to show that

$$
\sum \frac{b(n)}{\mu_{n}^{r-1}} \int_{x}^{\infty} I_{-1}\left(\mu_{n} t\right) f(t) d t
$$

tends to 0 as $x$ tends to $\infty$. The above becomes, after an integration by parts,

$$
\left.D_{0}(t) f(t)\right|_{x} ^{\infty}-\sum \frac{b(n)}{\mu_{n}^{r}} \int_{x}^{\infty} I_{0}\left(\mu_{n} t\right) f^{\prime}(t) d t
$$

The integration by parts is valid, and the integrated term tends to 0 as $x$ tends to $\infty$, because of our hypothesis on $D_{0}(x) f(x)$. After another integration by parts, the series in (3.8) becomes

$$
\left.D_{1}(t) f^{\prime}(t)\right|_{x} ^{\infty}-\sum \frac{b(n)}{\mu_{n}^{r+1}} \int_{x}^{\infty} I_{1}\left(\mu_{n} t\right) f^{\prime \prime}(t) d t .
$$


Now, $I_{1}(x)=O\left(x^{r / 2+1 / 4}\right)$ as $x$ tends to $\infty$. Hence,

$$
D_{1}(x)=O\left(x^{r / 2+1 / 4} \sum \frac{|b(n)|}{\mu_{n}^{r / 2+3 / 4}}\right)=O\left(x^{r / 2+1 / 4}\right)
$$

as $x$ tends to $\infty$, since $r / 2+3 / 4>\sigma_{a}^{*}$. Hence, the last integration by parts is valid, and the integrated term tends to 0 as $x$ tends to $\infty$, because of our hypothesis on $x^{r / 2+1 / 4} f^{\prime}(x)$. Using the above estimate for $I_{1}(x)$, we find that the modulus of the infinite series in (3.9) is less than

$$
A \sum \frac{|b(n)|}{\mu_{n}^{r / 2+3 / 4}} \int_{x}^{\infty} t^{r / 2+1 / 4}\left|f^{\prime \prime}(t)\right| d t=o(1)
$$

as $x$ tends to $\infty$, since both the series and integral converge.

\section{A Voronoï summation formula for functions of bounded variation.}

THEOREM 6. Let $f$ be of bounded variation on $[a, x]$, where $0<a<\lambda_{1}<x<\infty$. Then,

$$
\begin{aligned}
\frac{1}{2} \sum_{\lambda_{n} \leqq x}^{\prime} a(n)\left\{f\left(\lambda_{n}+0\right)+\right. & \left.f\left(\lambda_{n}-0\right)\right\} \\
& =\int_{a}^{x} Q_{0}^{\prime}(t) f(t) d t+\sum \frac{b(n)}{\mu_{n}^{r-1}} \int_{a}^{x} I_{-1}\left(\mu_{n} t\right) f(t) d t .
\end{aligned}
$$

Proof. The proof follows along the same lines as Landau's proof for $a(n)=r_{2}(n)$ [24, Satz 559]. We shall only indicate those details which differ from Landau's. As in [24], it suffices to show that

$$
\sum \frac{b(n)}{\mu_{n}^{r-1}} \int_{\alpha}^{\beta} I_{-1}\left(\mu_{n} t\right) f(t) d t+\int_{\alpha}^{\beta} Q_{0}^{\prime}(t) f(t) d t=0,
$$

where either

$$
\begin{aligned}
& \alpha=\lambda_{k}<\beta<\lambda_{k+1} \text {, and } f \text { is monotonically } \\
& \text { increasing on }\left[\lambda_{k}, \beta\right] \text { with } f\left(\lambda_{k}+0\right)=0,
\end{aligned}
$$

or

$$
\begin{aligned}
& \lambda_{k}<\alpha<\lambda_{k+1}=\beta \text {, and } f \text { is monotonically } \\
& \text { increasing on }\left[\alpha, \lambda_{k+1}\right] \text { with } f\left(\lambda_{k+1}-0\right)=0 \text {. }
\end{aligned}
$$

We shall prove (4.2) in the case (4.3). The proof for the case (4.4) is similar.

Now, $D_{0}(t)$ converges boundedly on $\left[\lambda_{k}, \beta\right]$. Thus, there exists a number $M_{1}>0$, depending upon the interval, such that, for $\lambda_{k} \leqq t \leqq \beta$ and every $m>0$,

$$
\left|\sum_{n=1}^{m} \frac{b(n)}{\mu_{n}^{r}} I_{0}\left(\mu_{n} t\right)\right|<M_{1} .
$$

Since $Q_{0}(t)$ is continuous, there exists a number $M_{2}>0$ such that, for $\lambda_{k} \leqq t \leqq \beta$,

$$
\left|Q_{0}(t)\right|<M_{2} \text {. }
$$


Let $\varepsilon>0$ be given. Choose $\delta>0$ such that $\lambda_{k}+\delta<\beta$ and

$$
f\left(\lambda_{k}+\delta\right)<\varepsilon / 8 M,
$$

where $M=\sup \left(M_{1}, M_{2}\right)$. Applying the second mean value theorem for integrals $[29$, p. 380], we find that

$$
\begin{aligned}
\int_{\lambda_{k}}^{\beta}\left\{\sum_{n=1}^{m} \frac{b(n)}{\mu_{n}^{r-1}} I_{-1}\left(\mu_{n} t\right)+Q_{0}^{\prime}(t)\right\} f(t) d t \\
=\left(\int_{\lambda_{k}}^{\lambda_{k}+\delta}+\int_{\lambda_{k}+\delta}^{\beta}\right)\left\{\sum_{n=1}^{m} \frac{b(n)}{\mu_{n}^{r-1}} I_{-1}\left(\mu_{n} t\right)+Q_{0}^{\prime}(t)\right\} f(t) d t \\
\quad=\left(f\left(\lambda_{k}+\delta\right) \int_{\xi_{1}(m)}^{\lambda_{k}+\delta}+f(\beta-0) \int_{\xi_{2}(m)}^{\beta}\right)\left\{\sum_{n=1}^{m} \frac{b(n)}{\mu_{n}^{r-1}} I_{-1}\left(\mu_{n} t\right)+Q_{0}^{\prime}(t)\right\} d t,
\end{aligned}
$$

where $\lambda_{k} \leqq \xi_{1}(m) \leqq \lambda_{k}+\delta \leqq \xi_{2}(m) \leqq \beta$. Upon integration and the use of (4.5)-(4.7), we have

$$
\left|f\left(\lambda_{k}+\delta\right) \int_{\xi_{1}(m)}^{\lambda_{k}+\delta}\left\{\sum_{n=1}^{m} \frac{b(n)}{\mu_{n}^{r-1}} I_{-1}\left(\mu_{n} t\right)+Q_{0}^{\prime}(t)\right\} d t\right|<\varepsilon\left(2 M_{1}+2 M_{2}\right) / 8 M \leqq \varepsilon / 2 .
$$

Now, $D_{0}(t)$ converges uniformly on $\left[\lambda_{k}+\delta, \beta\right]$, for $\left[\lambda_{k}+\delta, \beta\right]$ contains no members of $\left\{\lambda_{n}\right\}$. Clearly then, we can choose $m$ large enough so that

$$
\left|f(\beta-0)\left\{\sum_{n=1}^{m} \frac{b(n)}{\mu_{n}^{r}} I_{0}\left(\mu_{n} t\right)+Q_{0}(t)\right\}_{\xi_{2}(m)}^{\beta}\right|<\varepsilon / 2 .
$$

Using (4.9) and (4.10), we find from (4.8) that, for $m$ large enough,

$$
\left|\sum_{n=1}^{m} \frac{b(n)}{\mu_{n}^{r-1}} \int_{\lambda_{k}}^{\beta} I_{-1}\left(\mu_{n} t\right) f(t) d t+\int_{\dot{\lambda}_{k}}^{\beta} Q_{0}^{\prime}(t) f(t) d t\right|<\varepsilon
$$

from which (4.2) follows.

We now wish to extend the previous theorem to $a=0$. It is clear that we can use the same argument as in the above proof if $Q_{0}(t)$ is continuous at $t=0$ and if we can show that $D_{0}(t)$ converges boundedly on $0 \leqq t \leqq x$. Again, the Bessel functions $K_{v}$ and $Y_{v}$ cause difficulty because of their singularities at the origin. Thus, we consider only the case $\Delta(s)=\Gamma(s)$. In order to show the desired bounded convergence, we must derive an identity which is a generalization of a theorem of Landau [24, Satz 523] and of Theorem 2 in [2]. Our somewhat different proof enables us to establish the generalization.

We first state some properties of $J_{\nu}(x)$ that we shall need. For $\nu>0$ and $a>0$ $[32$, p. 406],

$$
\begin{aligned}
\int_{0}^{\infty} J_{v-1}(a t) J_{v}(t) d t & =a^{v-1}, & & a<1, \\
& =\frac{1}{2}, & & a=1, \\
& =0, & & a>1 .
\end{aligned}
$$


For arbitrary $\nu[32$, p. 45],

$$
(d / d x)\left\{x^{-v} J_{v}(x)\right\}=-x^{-v} J_{v+1}(x) .
$$

Instead of introducing additional notation, we keep the same notation, $A_{q}(x)$, $Q_{q}(x)$ and $D_{q}(x)$, although the roles of $\varphi$ and $\psi$ are reversed, in the following theorem.

TheOREM 7. Let $\Delta(s)=\Gamma(s)$. Suppose that $r>-2$ and that the poles of $\psi$ lie in the half-plane $\sigma>0$. Then, for $x \geqq 0$ and $y>0$,

$\sum_{\mu_{n} \leqq y} b(n)\left(x / \mu_{n}\right)^{r / 2} J_{r}\left(2\left\{\mu_{n} x\right\}^{1 / 2}\right)$

$$
\begin{aligned}
= & \sum_{\lambda_{n} \leqq x}^{\prime} a(n)-\sum a(n)\left(x / \lambda_{n}\right)^{(r+1) / 2} \int_{2(x y)^{1 / 2}}^{\infty} J_{r+1}\left(t\left\{\lambda_{n} / x\right\}^{1 / 2}\right) J_{r+2}(t) d t \\
& +(x / y)^{(r+1) / 2} J_{r+1}\left(2\{x y\}^{1 / 2}\right) D_{1}(y)+(x / y)^{r / 2} J_{r}\left(2\{x y\}^{1 / 2}\right) D_{0}(y) \\
& -\left.(x / t)^{r / 2} J_{r}\left(2\{x t\}^{1 / 2}\right) D_{0}(t)\right|_{t=0} \\
& +\int_{0}^{y}(x / t)^{r / 2} J_{r}\left(2\{x t\}^{1 / 2}\right) Q_{0}^{\prime}(t) d t
\end{aligned}
$$

The hypothesis concerning the poles is needed for technical reasons which are pointed out in the proof.

Proof. By (4.11) for $r>-2$,

$$
\begin{aligned}
\sum_{\lambda_{n} \leqq x}^{\prime} a(n)= & \sum a(n)\left(x / \lambda_{n}\right)^{(r+1) / 2} \int_{0}^{\infty} J_{r+1}\left(t\left\{\lambda_{n} / x\right\}^{1 / 2}\right) J_{r+2}(t) d t \\
= & \sum a(n)\left(x / \lambda_{n}\right)^{(r+1) / 2} \\
& \times\left(\int_{0}^{2(x y)^{1 / 2}}+\int_{2(x y)^{1 / 2}}^{\infty}\right) J_{r+1}\left(t\left\{\lambda_{n} / x\right\}^{1 / 2}\right) J_{r+2}(t) d t .
\end{aligned}
$$

Replacing $t$ by $(2 x t)^{1 / 2}$ in the first integral on the right-hand side of (4.14), we obtain

$$
\begin{aligned}
\sum a(n)\left(x / \lambda_{n}\right)^{(r+1) / 2} \int_{0}^{2(x y)^{1 / 2}} J_{r+1}\left(t\left\{\lambda_{n} / x\right\}^{1 / 2}\right) J_{r+2}(t) d t \\
=\int_{0}^{y}(x / t)^{1+r / 2} J_{r+2}\left(2\{x t\}^{1 / 2}\right) \sum a(n)\left(t / \lambda_{n}\right)^{(r+1) / 2} J_{r+1}\left(2\left\{\lambda_{n} t\right\}^{1 / 2}\right) d t \\
=\int_{0}^{y}(x / t)^{1+r / 2} J_{r+2}\left(2\{x t\}^{1 / 2}\right) D_{1}(t) d t
\end{aligned}
$$

where the inversion in order of summation and integration is justified since $D_{1}(t)$ converges absolutely. Upon an integration by parts with the aid of (4.12), the above integral becomes

$$
-(x / y)^{(r+1) / 2} J_{r+1}\left(2\{x y\}^{1 / 2}\right) D_{1}(y)+\int_{0}^{y}(x / t)^{(r+1) / 2} J_{r+1}\left(2\{x t\}^{1 / 2}\right) D_{0}(t) d t,
$$


since $D_{1}(0)=0$, as the poles of $\psi(s)$ are in $\sigma>0$. Upon a similar integration by parts, this last integral becomes

$$
\begin{aligned}
-(x / y)^{r / 2} J_{r}\left(2\{x y\}^{1 / 2}\right) D_{0}(y) & +\left.(x / t)^{r / 2} J_{r}\left(2\{x t\}^{1 / 2}\right) D_{0}(t)\right|_{t=0} \\
& +\int_{0}^{y}(x / t)^{r / 2} J_{r}\left(2\{x t\}^{1 / 2}\right) d D_{0}(t) .
\end{aligned}
$$

Note that $D_{0}(0)$ is finite since the poles of $\psi$ lie in the right half-plane. The integral in (4.17) can be written as

$$
\begin{aligned}
\int_{0}^{y}(x / t)^{r / 2} J_{r}\left(2\{x t\}^{1 / 2}\right)\left\{d A_{0}(t)-Q_{0}^{\prime}(t) d t\right\} & \\
& =\sum_{\mu_{n} \leqq y} b(n)\left(x / \mu_{n}\right)^{r / 2} J_{r}\left(2\left\{\mu_{n} x\right\}^{1 / 2}\right)-\int_{0}^{y}(x / t)^{r / 2} J_{r}\left(2\{x t\}^{1 / 2}\right) Q_{0}^{\prime}(t) d t .
\end{aligned}
$$

Combining (4.14)-(4.18), we arrive at (4.13).

COROLlARY 8. In addition to the notation and hypotheses of Theorem 7, suppose that $r \geqq 0$ and that $D_{0}(x)=O\left(x^{r / 2}\right)$ as $x$ tends to $\infty$. Then,

$$
\sum b(n)\left(x / \mu_{n}\right)^{r / 2} J_{r}\left(2\left\{\mu_{n} x\right\}^{1 / 2}\right)
$$

converges boundedly on any compact interval in $x \geqq 0$.

Proof. In view of Theorem 7, we only need to show that all expressions on the right-hand side of (4.13) are $O(1)$ as $y$ tends to $\infty$. Also, in view of the theorem of Chandrasekharan and Narasimhan stated in $\S 2$, we may assume that $0 \leqq x \leqq \varepsilon$, where $\varepsilon>0$ is fixed.

The first expression is obviously $O(1)$. The second term is shown to be $O(1)$ in [2]. From (3.10), $D_{1}(y)=O\left(y^{r / 2+1 / 4}\right)$ as $y$ tends to $\infty$. Since $r \geqq 0, J_{r+1}\left(2\{x y\}^{1 / 2}\right)$ is bounded for all $x y \geqq 0$. It follows that the third term is $O(1)$. Since $r \geqq 0, J_{r}\left(2\{x y\}^{1 / 2}\right)$ is bounded. Since also $D_{0}(y)=O\left(y^{r / 2}\right)$, we see that the fourth term is $O(1)$. The fifth term is clearly $O(1)$.

Lastly, we consider the integral. It will be sufficient to show that

$$
\int_{a}^{\infty} t^{-r / 2} J_{r}\left(2\{x t\}^{1 / 2}\right) Q_{0}^{\prime}(t) d t
$$

converges, where $a>0$. Since $\psi$ has only poles, $Q_{0}(t)$ is a finite sum of products of powers of $t$ and of $\log t$. For $t$ large, the largest term will be no greater than a multiple of $t^{\sigma_{a}^{*}} \log ^{k} t$ for some nonnegative integer $k$. Now, as $t$ tends to $\infty[32$, p. 199],

$$
J_{v}(t)=\left(c_{1} e^{i t}+c_{2} e^{-i t}\right) t^{-1 / 2}+\left(c_{3} e^{i t}+c_{4} e^{-i t}\right) t^{-3 / 2}+O\left(t^{-5 / 2}\right),
$$

where $c_{1}, \ldots, c_{4}$ are constants. Thus, (4.19) converges if and only if

$$
\int_{a}^{\infty} e^{i t} t^{2 \sigma_{a}^{*}-r-3 / 2} \log ^{k} t d t
$$


converges. But since $2 \sigma_{a}^{*}-r-3 / 2<0$, the above integral converges. Hence, the last term on the right-hand side of (4.13) is $O(1)$ as $y$ tends to $\infty$, and the proof is complete.

In light of our comments after Theorem 6, we now have

THEOREM 9. Suppose that the poles of $\varphi(s)$ are in the half-plane $\sigma>0$. Under the hypotheses of Theorem 6 and Corollary 8, (4.1) is valid with $a=0$.

We now wish to extend Theorem 6 by letting $x=\infty$.

THEOREM 10. Let $f$ be of bounded variation on $[a, x]$ for every $x, 0<a<\lambda_{1}<x<\infty$, and suppose that $f$ is the integral of $f^{\prime}$. Assume that $D_{0}(x) f(x)$ tends to 0 as $x$ tends to $\infty$,

$$
\int_{x}^{\infty} t^{\sigma_{a}^{*}+\varepsilon-1 / 2}\left|f^{\prime}(t)\right| d t<\infty
$$

for some $\varepsilon>0$, and

$$
\int_{x}^{\infty} D_{0}(t) f^{\prime}(t) d t<\infty
$$

Then,

$$
\begin{gathered}
\lim _{x \rightarrow \infty}\left(\frac{1}{2} \sum_{\lambda_{n} \leqq x}^{\prime} a(n)\left\{f\left(\lambda_{n}+0\right)+f\left(\lambda_{n}-0\right)\right\}-\int_{a}^{x} Q_{0}^{\prime}(t) f(t) d t\right) \\
=\sum \frac{b(n)}{\mu_{n}^{r-1}} \int_{a}^{\infty} I_{-1}\left(\mu_{n} t\right) f(t) d t,
\end{gathered}
$$

provided that the series on the right-hand side converges.

Proof. We proceed as at the beginning of the proof of Theorem 5. After an integration by parts, we see that it is sufficient to show that

$$
\sum \frac{b(n)}{\mu_{n}^{r}} \int_{x}^{\infty} I_{0}\left(\mu_{n} t\right) f^{\prime}(t) d t
$$

tends to 0 as $x$ tends to $\infty$.

Write the above as

$$
\left(\sum_{\mu_{n} \leqq x}+\sum_{\mu_{n}>x}\right) \frac{b(n)}{\mu_{n}^{r}} \int_{x}^{\infty} I_{0}\left(\mu_{n} t\right) f^{\prime}(t) d t=S_{1}+S_{2},
$$

say. In the sum $S_{1}, \mu_{n} \leqq t$. Since also $\sigma_{a}^{*} \geqq r / 2+1 / 4$ [7, p. 111],

$$
\begin{aligned}
\left|S_{1}\right| & \leqq A \sum_{\mu_{n} \leqq x} \frac{|b(n)|}{\mu_{n}^{r / 2+1 / 4}} \int_{x}^{\infty} t^{r / 2-1 / 4}\left|f^{\prime}(t)\right| d t \\
& \leqq A \sum_{\mu_{n} \leqq x} \frac{|b(n)|}{\mu_{n}^{\sigma_{a}^{*}+8}} \int_{x}^{\infty} t^{\sigma_{a}^{*}+\varepsilon-1 / 2}\left|f^{\prime}(t)\right| d t=o(1)
\end{aligned}
$$

as $x$ tends to $\infty$, by $(4.20)$. 
To estimate $S_{2}$ we regard $x$ as fixed for the moment. Since the series on the right-hand side of (4.22) converges, given $\varepsilon>0$, there exists an $x_{0}$ such that, for $x^{\prime} \geqq x_{0}$,

$$
\left|\sum_{\mu_{n}>x^{\prime}} \frac{b(n)}{\mu_{n}^{r}} \int_{x}^{\infty} I_{0}\left(\mu_{n} t\right) f^{\prime}(t) d t\right|<\varepsilon
$$

If $x_{0} \leqq x$, in particular we can take $x^{\prime}=x$. Thus, $\left|S_{2}\right|<\varepsilon$, and we are done. If $x_{0}>x$, there remains to estimate

$$
\sum_{x<\mu_{n} \leqq x_{0}} \frac{b(n)}{\mu_{n}^{r}} \int_{x}^{\infty} I_{0}\left(\mu_{n} t\right) f^{\prime}(t) d t=\sum_{x<\mu_{n} \leqq x_{0}} \frac{b(n)}{\mu_{n}^{r}}\left(\int_{x}^{\mu_{n}}+\int_{\mu_{n}}^{\infty}\right) I_{0}\left(\mu_{n} t\right) f^{\prime}(t) d t=S_{3}+S_{4},
$$

say. $S_{4}$ is estimated in the same way as $S_{1}$, and we easily obtain

$$
\left|S_{4}\right| \leqq A \sum_{\mu_{n}>x} \frac{|b(n)|}{\mu_{n}^{\sigma_{a}^{*}+\varepsilon}} \int_{x}^{\infty} t^{\sigma_{a}^{*}+\varepsilon-1 / 2}\left|f^{\prime}(t)\right| d t=o(1)
$$

as $x$ tends to $\infty$. Now,

$$
\begin{aligned}
S_{3} & =\int_{x}^{x_{0}} \sum_{\mu_{n} \geqq t} \frac{b(n)}{\mu_{n}^{r}} I_{0}\left(\mu_{n} t\right) f^{\prime}(t) d t \\
& =\int_{x}^{x_{0}}\left\{D_{0}(t)-\sum_{\mu_{n}<t} \frac{b(n)}{\mu_{n}^{r}} I_{0}\left(\mu_{n} t\right)\right\} f^{\prime}(t) d t .
\end{aligned}
$$

By (4.21), the integral involving $D_{0}(t)$ is $o(1)$ as $x$ tends to $\infty$. Now,

$$
\left|\sum_{\mu_{n}<t} \frac{b(n)}{\mu_{n}^{r}} I_{0}\left(\mu_{n} t\right)\right| \leqq A t^{r / 2-1 / 4} \sum_{\mu_{n}<t} \frac{|b(n)|}{\mu_{n}^{r / 2+1 / 4}} \leqq A t^{\sigma_{a}^{*}+\varepsilon-1 / 2}
$$

by partial summation. Thus, by (4.20), the integral of the sum on the right-hand side of (4.23) is $o(1)$ as $x$ tends to $\infty$. This completes the proof.

5. Additional remarks on the theorems. We remark that Wilton [34] has stated a theorem similar to Theorem 10 when $a(n)=d(n)$. The difference in the two theorems is that Wilton has the assumption that $f(x)$ tends to 0 , while we have the hypothesis that $D_{0}(x) f(x)$ tends to 0 as $x$ tends to $\infty$. Dixon and Ferrar [12] restate Wilton's theorem with the hypothesis that $x^{1 / 2+\varepsilon} f(x)$ tends to 0 as $x$ tends to $\infty$ for some $\varepsilon>0$. In a footnote they remark that “... his (Wilton's) statement seems, to us, to contain a misprint". Since $D_{0}(x)=O\left(x^{\theta}\right)$ for some $\theta, 1 / 4<\theta<1 / 3$, our hypothesis lies "between" those of Wilton and Dixon and Ferrar.

The key to proving Theorem 9 is identity (4.13). Wilton [33] has proven a similar, but even more complicated, identity when $\Delta(s)=\Gamma^{2}\left(\frac{1}{2} s\right)$ and $a(n)=d(n)$. This identity was the essential ingredient in extending the Voronoï formula to $a=0$ in [34].

We have indicated several references to the literature for summation formulae involving $d(n)$ and $r_{2}(n)$. We indicate a few additional arithmetical functions that are covered by our theorems and have been discussed in the literature. Let $\left\{\lambda_{n}\right\}$ 
be the values assumed by a positive definite quadratic form in two variables. Voronoï [31] first gave the summation formulae here. It is also proved in [22] and [2]. Let $\{a(n)\}$ be the coefficients of a modular cusp form of negative dimension. Then results are given in [22], [14], [20] and [2]. Let $a(n)=F(n)$, the ideal function for a quadratic number field $K$. If $K$ is imaginary, results are given in [22], [8] and [2]. If $K$ is real, results are given in [8]. We remark that the proof of Theorem 3 in [8], when $K$ is real, is not correct, because the estimate $I_{1}(t)=O\left(t^{3 / 2}\right)$ as $t$ tends to 0 , given on p. 308 , is false.

We have not discussed here Voronoï summation formulae where the infinite series do not converge in the ordinary sense, but are summable by Riesz means [10], [11], [18] and [25]. In particular, such formulae arise when (2.2) does not hold for $q=0$. It would be desirable to have convergent Voronoï type summation formulae for such arithmetical functions. One might hope then to find a general formula for $\sum_{\lambda_{n} \leqq x} a(n) f\left(\lambda_{n}\right)\left(x-\lambda_{n}\right)^{q}$ for suitable $q>0$, but no such formula appears to have been discovered.

6. Applications. Wilton [35] has given some applications of the Voronoï formula in estimating sums of the form $\sum_{n \leqq x} d(n) f(n)$. Several authors have derived arithmetical identities by using particular examples of $f(x)$. We give several identities here in the case $\Delta(s)=\Gamma(s)$. As is to be expected, several of our identities hold in a more general setting. For each example we refer to theorems which insure the validity of our application, but in each case there is more than one choice of theorems possible.

We shall need the values of several integrals.

For $a, p>0$ and $\nu>-1$ [32, p. 394],

$$
\int_{0}^{\infty} t^{v+1} J_{v}(a t) \exp \left(-p^{2} t^{2}\right) d t=\frac{a^{v}}{\left(2 p^{2}\right)^{v+1}} \exp \left(\frac{-a^{2}}{4 p^{2}}\right) .
$$

For $a, b>0$ and $\nu>-1[32$, p. 386],

$$
\int_{0}^{\infty} t^{\nu+1} J_{v}(b t) e^{-a t} d t=\frac{2 a(2 b)^{v} \Gamma(\nu+3 / 2)}{\left(a^{2}+b^{2}\right)^{v+3 / 2} \pi^{1 / 2}}
$$

For $a, b>0$ and $\nu>-\frac{1}{2}[32$, p. 386$]$,

$$
\int_{0}^{\infty} t^{v} J_{v}(b t) e^{-a t} d t=\frac{(2 b)^{v} \Gamma\left(\nu+\frac{1}{2}\right)}{\left(a^{2}+b^{2}\right)^{v+1 / 2} \pi^{1 / 2}}
$$

For $\operatorname{Re} \mu>|\operatorname{Re} \nu|[32$, p. 388],

$$
\int_{0}^{\infty} t^{\mu-1} K_{v}(t) d t=2^{\mu-2} \Gamma\left(\frac{1}{2}\{\mu-\nu\}\right) \Gamma\left(\frac{1}{2}\{\mu+\nu\}\right) .
$$

For $a, b, \operatorname{Re} \mu>0$ and $\nu>-1[32$, p. 410],

$$
\int_{0}^{\infty} K_{\mu}(a t) J_{v}(b t) t^{\mu+v+1} d t=\frac{(2 a)^{\mu}(2 b)^{\nu} \Gamma(\mu+\nu+1)}{\left(a^{2}+b^{2}\right)^{\mu+v+1}} .
$$


For $a>0, z$ real, $\operatorname{Re} \mu>-1$, and $\nu$ complex [32, p. 417],

$$
\int_{0}^{\infty} \frac{K_{v}\left(a\left\{t^{2}+z^{2}\right\}^{1 / 2}\right)}{\left(t^{2}+z^{2}\right)^{v / 2}} t^{2 \mu+1} d t=\frac{2^{\mu} \Gamma(\mu+1)}{a^{\mu+1} z^{\nu-\mu-1}} K_{v-\mu-1}(a z) .
$$

For $a, b>0, z \neq 0$ and real, $\mu>-1$, and $\nu$ complex [32, p. 416],

$$
\begin{aligned}
\int_{0}^{\infty} J_{\mu}(b t) \frac{K_{v}\left(a\left\{t^{2}+z^{2}\right\}^{1 / 2}\right)}{\left(t^{2}+z^{2}\right)^{\nu / 2}} t^{\mu+1} d t \\
=\left(b^{\mu} / a^{\nu}\right)\left(\left\{a^{2}+b^{2}\right\}^{1 / 2} / z\right)^{\nu-\mu-1} K_{\nu-\mu-1}\left(z\left\{a^{2}+b^{2}\right\}^{1 / 2}\right)
\end{aligned}
$$

For $a, b, p>0$ and $\nu>-1[32$, p. 395],

$$
\int_{0}^{\infty} \exp \left(-p^{2} t^{2}\right) J_{v}(a t) J_{v}(b t) t d t=\frac{1}{2 p^{2}} \exp \left(-\frac{a^{2}+b^{2}}{4 p^{2}}\right) I_{v}\left(\frac{a b}{2 p^{2}}\right),
$$

where here $I_{v}$ is the Bessel function defined in [32, p. 77]. For $a, b>0, n>0$ and integral, and $n+\nu>-1[15$, p. 721$]$,

$$
\int_{0}^{\infty} t^{n+v / 2} e^{-a t} J_{v}\left(2 b t^{1 / 2}\right) d t=n ! b^{v} \exp \left(-b^{2} / a\right) a^{-n-v-1} L_{n}^{(v)}\left(b^{2} / a\right),
$$

where $L_{n}^{(v)}(x)$ is the Laguerre polynomial defined by [15, p. 1037]

$$
L_{n}^{(v)}(x)=\frac{1}{n !} e^{x} x^{-v}\left(d^{n} / d x^{n}\right)\left(e^{-x} x^{n+v}\right) .
$$

EXAmPLE 1. Let $f(t)=e^{-t y}, y>0$. Assume the hypotheses of Theorems 3 and 5. An easy calculation gives

$$
\int_{0}^{\infty} Q_{0}^{\prime}(t) e^{-t y} d t=\frac{1}{2 \pi i} \int_{C_{0}} \Gamma(s) \varphi(s) y^{-s} d s=P(y),
$$

say. Replacing $t$ by $t^{2}$ in the second expression on the right side of (3.1) and using (6.1), we have, for $r>0$,

$$
\int_{0}^{\infty} I_{-1}\left(\mu_{n} t\right) e^{-t y} d t=\mu_{n}^{r-1} \exp \left(-\mu_{n} / y\right) / y^{r}
$$

Thus, we have the "modular relation"

$$
\sum a(n) e^{-\lambda_{n} y}=y^{-r} \sum b(n) \exp \left(-\mu_{n} / y\right)+P(y),
$$

which is, in fact, equivalent to the functional equation [6, Theorem 1].

EXAMPLE 2. Let $f(t)=\exp \left(-t^{1 / 2} y\right), y>0$. Assume the hypotheses of Theorems 2, 5 and 7 and Corollary 8. Using the same substitution as in Example 1, we have by (6.2), for $r>0$,

$$
\int_{0}^{\infty} I_{-1}\left(\mu_{n} t\right) \exp \left(-t^{1 / 2} y\right) d t=\frac{4^{r} \mu_{n}^{r-1} y \Gamma\left(r+\frac{1}{2}\right)}{\left(y^{2}+4 \mu_{n}\right)^{r+1 / 2} \pi^{1 / 2}} .
$$


Thus, we have the following identity that has been very useful in obtaining $\Omega$ results for the average order of certain arithmetical functions:

$$
\begin{aligned}
& \sum a(n) \exp \left(-\lambda_{n}^{1 / 2} y\right) \\
& =\frac{1}{\pi i} \int_{C_{0}} \Gamma(2 s) \varphi(s) y^{-2 s} d s+\frac{4^{r} y \Gamma\left(r+\frac{1}{2}\right)}{\pi^{1 / 2}} \sum \frac{b(n)}{\left(y^{2}+4 \mu_{n}\right)^{r+1 / 2}} .
\end{aligned}
$$

This identity was first discovered by Hardy for the special cases $r_{2}(n)$ and $\tau(n)$, Ramanujan's arithmetical function (see the examples in [6]). Hamburger [21] derived a special case of (6.10) when $r=\frac{1}{2}$. A more general identity is given in [6, Lemma 6].

EXAMPLE 3. Let $f(t)=t^{-1 / 2} \exp \left(-t^{1 / 2} y\right), y>0$. This next identity can be obtained by formally integrating (6.10). However, if we assume the hypotheses of Theorems 2, 5 and 7 and Corollary 8, we have upon the use of (6.3), for $r>\frac{1}{2}$,

$$
\begin{aligned}
& \sum a(n) \lambda_{n}^{-1 / 2} \exp \left(-\lambda_{n}^{1 / 2} y\right) \\
& \quad=\frac{1}{\pi i} \int_{C_{0}} \Gamma(2 s-1) \varphi(s) y^{-2 s+1} d s+\frac{4^{r} \Gamma\left(r-\frac{1}{2}\right)}{2 \pi^{1 / 2}} \sum \frac{b(n)}{\left(y^{2}+4 \mu_{n}\right)^{r-1 / 2}} .
\end{aligned}
$$

EXAMPLE 4. Let $f(t)=\log ^{q}(x / t)$, where $q$ is a positive integer. Assume the hypotheses of Theorems 2 and 7 and Corollary 8 . Upon $q$ integrations by parts,

$$
\int_{0}^{x} Q_{0}^{\prime}(t) \log ^{q}(x / t) d t=\frac{q !}{2 \pi i} \int_{C_{0}} \frac{\varphi(s) x^{s}}{s^{q+1}} d s=q ! R(x ; q),
$$

say. Replacing $t$ by $2\left(\mu_{n} t\right)^{1 / 2}$ and integrating by parts once with the use of the formula [32, p. 45], $(d / d x)\left\{x^{v} J_{v}(x)\right\}=x^{v} J_{v-1}(x)$, we find that, for $r>0$,

$$
\int_{0}^{x}\left(\mu_{n} t\right)^{(r-1) / 2} J_{r-1}\left(2\left\{\mu_{n} t\right\}^{1 / 2}\right) \log ^{q}(x / t) d t=\frac{2^{q-r} q}{\mu_{n}} \int_{0}^{\xi} t^{r-1} J_{r}(t) \log ^{q-1}(\xi / t) d t,
$$

where $\xi=2\left(\mu_{n} x\right)^{1 / 2}$. We thus have

$$
\frac{1}{q !} \sum_{\lambda_{n} \leqq x}^{\prime} a(n) \log ^{q}\left(x / \lambda_{n}\right)=R(x ; q)+\frac{2^{q-r}}{(q-1) !} \sum \frac{b(n)}{\mu_{n}^{r}} \int_{0}^{\xi} t^{r-1} J_{r}(t) \log ^{q-1}(\xi / t) d t,
$$

an identity proved by us in [4, Theorem 3].

EXAMPLE 5. Let $f(t)=t^{(s-r) / 2} K_{s-r}\left(2\{t y\}^{1 / 2}\right)$, where $\sigma>r$ and $y>0$. Assume the hypotheses of Theorems 2 and 10. With the use of (6.4), a straightforward calculation using the same substitution as in Theorem 1 yields

$$
\int_{0}^{\infty} Q_{0}^{\prime}(t) t^{(s-r) / 2} K_{s-r}\left(2\{t y\}^{1 / 2}\right) d t=\frac{1}{4 \pi i} \int_{C_{0}} \Gamma(z) \Gamma(z+s-r) \varphi(z) y^{-z-(s-r) / 2} d z .
$$

With the use of (6.5), we obtain, for $r>0$,

$$
\int_{0}^{\infty} I_{-1}\left(\mu_{n} t\right) f(t) d t=\frac{\mu_{n}^{r-1} y^{(s-r) / 2} \Gamma(s)}{2\left(\mu_{n}+y\right)^{s}} .
$$


We thus obtain for $\sigma>r$,

$$
\begin{aligned}
\Gamma(s) \sum \frac{b(n)}{\left(\mu_{n}+y\right)^{s}}= & 2 \sum a(n)\left(\lambda_{n} / y\right)^{(s-r) / 2} K_{s-r}\left(2\left\{\lambda_{n} y\right\}^{1 / 2}\right) \\
& -\frac{1}{2 \pi i} \int_{C_{0}} \Gamma(z) \Gamma(z+s-r) \varphi(z) y^{-z-s+r} d z .
\end{aligned}
$$

With the roles of $\varphi$ and $\psi$ reversed, this identity for the "generalized" Dirichlet series on the left was first given by us in [1]. Another proof is given in [5]. An equivalent identity when $a(n)=r_{2}(n)$ is given in [10]. (6.11) may be used to give an analytic continuation of the "generalized" Dirichlet series.

EXAMPLE 6. Let $f(t)=(b /(t+a))^{v / 2} K_{v}\left(2\{(t+a) b\}^{1 / 2}\right)$, where $a, b>0$ and $v$ is arbitrary. Assume the hypotheses of Theorems 3 and 10. Replacing $t$ by $t^{2}$ and using (6.6), we have

$$
\int_{0}^{\infty} Q_{0}^{\prime}(t) f(t) d t=\frac{1}{2 \pi i} \int_{C_{0}} \Gamma(s) \varphi(s)(b / a)^{(v-s) / 2} K_{v-s}\left(2\{a b\}^{1 / 2}\right)=U(a, b),
$$

say. By (6.7) for $r>0$,

$$
\int_{0}^{\infty} I_{-1}\left(\mu_{n} t\right) f(t) d t=\mu_{n}^{r-1}\left(\left(\mu_{n}+b\right) / a\right)^{(v-r) / 2} K_{v-r}\left(2\left\{\left(\mu_{n}+b\right) a\right\}^{1 / 2}\right) .
$$

Since $K_{v}(z)=K_{-v}(z)$, we thus obtain

$$
\begin{aligned}
\sum a(n)\left(b /\left(\lambda_{n}+a\right)\right)^{v / 2} K_{v}\left(2\left\{\left(\lambda_{n}+a\right) b\right\}^{1 / 2}\right) & \\
= & U(a, b)+\sum b(n)\left(a /\left(\mu_{n}+b\right)\right)^{(r-v) / 2} K_{r-v}\left(2\left\{\left(\mu_{n}+b\right) a\right\}^{1 / 2}\right),
\end{aligned}
$$

an identity obtained by us in [5, Theorem 9.1]. There, we did not evaluate $U(a, b)$.

EXAMPLE 7. Let $f(t)=e^{-t y} t^{-(r-1) / 2} J_{r-1}\left(a t^{1 / 2}\right)$, where $a, r, y>0$. Assume the hypotheses of Theorems 2 and 10. By (6.8),

$$
\int_{0}^{\infty} I_{-1}\left(\mu_{n} t\right) f(t) d t=\frac{\mu_{n}^{(r-1) / 2}}{y} \exp \left(-\frac{a^{2}+4 \mu_{n}}{4 y}\right) I_{r-1}\left(\frac{a \mu_{n}^{1 / 2}}{y}\right),
$$

where $I_{r-1}$ is a Bessel function. Hence,

$$
\begin{aligned}
\sum a(n) \exp \left(-\lambda_{n} y\right) \lambda_{n}^{-(r-1) / 2} J_{r-1}\left(a \lambda_{n}^{1 / 2}\right) & \\
= & \int_{0}^{\infty} Q_{0}^{\prime}(t) e^{-t y} t^{-(r-1) / 2} J_{r-1}\left(a t^{1 / 2}\right) d t \\
& +y^{-1} \sum b(n) \exp \left(-\frac{a^{2}+4 \mu_{n}}{4 y}\right) \mu_{n}^{-(r-1) / 2} I_{r-1}\left(\frac{a \mu_{n}^{1 / 2}}{y}\right),
\end{aligned}
$$

an identity which appears to be new. Ordinarily, the integral in (6.12) cannot be explicitly evaluated. However, if $\varphi$ has at most one pole, that at $s=r$ with residue $\rho$, which is the case in most examples, the integral reduces to

$$
\left(2 \rho a^{r-1} /(2 y)^{r}\right) \exp \left(-a^{2} / 4 y\right),
$$


by (6.1). If, in addition, $r=1,(6.12)$ reduces to

$$
\begin{aligned}
y \sum a(n) \exp \left(-\lambda_{n} y\right) & J_{0}\left(a \lambda_{n}^{1 / 2}\right) \\
= & \rho \exp \left(-a^{2} / 4 y\right)+\sum b(n) \exp \left(-\frac{a^{2}+4 \mu_{n}}{4 y}\right) I_{0}\left(\frac{a \mu_{n}^{1 / 2}}{y}\right) .
\end{aligned}
$$

EXAMPLE 8. Let $f(t)=t^{m} e^{-t y}$, where $y>0$ and $m$ is a positive integer. Assume the hypotheses of Theorems 3 and 10. By (6.9) if $m+r>0$,

$$
\int_{0}^{\infty} I_{-1}\left(\mu_{n} t\right) t^{m} e^{-t y} d t=m ! \mu_{n}^{r-1} \exp \left(-\mu_{n} / y\right) y^{-m-r} L_{m}^{(r-1)}\left(\mu_{n} / y\right)
$$

Hence, we have

$$
\begin{aligned}
\sum a(n) \lambda_{n}^{m} \exp \left(-\lambda_{n} y\right) & \\
= & \frac{1}{2 \pi i} \int_{C_{0}} \Gamma(s+m) \varphi(s) y^{-s-m} d s+m ! y^{-m-r} \sum b(n) \exp \left(-\mu_{n} / y\right) L_{m}^{(r-1)}\left(\mu_{n} / y\right),
\end{aligned}
$$

an identity we derived in [5, Theorem 10.1].

\section{REFERENCES}

1. B. C. Berndt, Generalised Dirichlet series and Hecke's functional equation, Proc. Edinburgh Math. Soc. (2) 15 (1966/67), 309-313. MR 37 \#1325.

2. - Arithmetical identities and Hecke's functional equation, Proc. Edinburgh Math Soc. (2) 16 (1968/69), 221-226. MR 40 \#4212.

3. - Identities involving the coefficients of a class of Dirichlet series. I, Trans. Amer. Math. Soc. 137 (1969), 345-359. MR 38 \#4656.

4. - Identities involving the coefficients of a class of Dirichlet series. II, Trans. Amer. Math. Soc. 137 (1969), 361-374. MR 38 \#4656.

5. - Identities involving the coefficients of a class of Dirichlet series. III, Trans. Amer. Math. Soc. 146 (1969), 323-348. MR 40 \#5551.

6. K. Chandrasekharan and Raghavan Narasimhan, Hecke's functional equation and arithmetical identities, Ann. of Math. (2) 74 (1961), 1-23. MR 30 \#1988.

7. - Functional equations with multiple gamma factors and the average order of arithmetical functions, Ann. of Math. (2) 76 (1962), 93-136. MR 25 \#3911.

8. - An approximate reciprocity formula for some exponential sums, Comment. Math. Helv. 43 (1968), 296-310. MR 40 \#2617.

9. A. L. Dixon and W. L. Ferrar, Lattice-point summation formulae, Quart. J. Math. Oxford Ser. 2 (1931), 31-54.

10. - Some summations over the lattice points of a circle. I, Quart. J. Math. Oxford Ser. 5 (1934), 48-63.

11. - Some summations over the lattice points of a circle. II, Quart. J. Math. Oxford Ser. 5 (1934), 172-185.

12. - On the summation formulae of Voronoi and Poisson, Quart. J. Math. Oxford Ser. 8 (1937), 66-74.

13. W. L. Ferrar, Summation formulae and their relation to Dirichlet's series, Compositio Math. 1 (1935), 344-360.

14. - Summation formulae and their relation to Dirichlet's series. II, Compositio Math. 4 (1937), 394-405. 
15. I. S. Gradšteĭn and I. M. Ryžik, Tables of integrals, series and products, 4 th ed., Fizmatgiz, Moscow, 1963; English transl., Academic Press, New York, 1965. MR 28 \#5198; MR 33 \#5952.

16. A. P. Guinand, A class of self-reciprocal functions connected with summation formulae, Proc. London Math. Soc. (2) 43 (1937), 439-448.

17. - Summation formulae and self-reciprocal functions, Quart. J. Math. Oxford Ser. 9 (1938), 53-67.

18. - Summation formulae and self-reciprocal functions. II, Quart. J. Math. Oxford Ser. 10 (1939), 104-118.

19. — - Finite summation formulae, Quart. J. Math. Oxford Ser. 10 (1939), 38-44.

20. - Integral modular forms and summation formulae, Proc. Cambridge Philos. Soc. 43 (1947), 127-129. MR 8, 198.

21. H. Hamburger, Über einige Beziehungen, die mit der Funktionalgleichung der Riemannschen $\zeta$-Funktion äquivalent sind, Math. Ann. 85 (1922), 129-140.

22. N. S. Koshliakov, Application of the theory of sum-formulae to the investigation of a class of one-valued analytical functions in the theory of numbers, Messenger Math. 58 (1929), 1-23.

23. ——, On Voronoi's sum-formula, Messenger Math. 58 (1929), 30-32.

24. E. Landau, Vorlesungen über Zahlentheorie, Zweiter Band, Chelsea, New York, 1947.

25. C. Nasim, A summation formula involving $\sigma_{k}(n), k>1$, Canad. J. Math. 21 (1969), 951-964. MR 40 \#110.

26. - On the summation formula of Voronoi, Trans. Amer. Math. Soc. (to appear).

27. T. L. Pearson, Note on the Hardy-Landau summation formula, Canad. Math. Bull. 8 (1965), 717-720. MR 33 \#2616.

28. W. Sierpiński, O pewnem zagadnieniu z rachunku funkcyj asymptotycznych, Prace Math.Fiz. 17 (1906), 77-118.

29. E. C. Titchmarsh, The theory of functions, 2nd ed., Clarendon Press, Oxford, 1939.

30. M. G. Voronoĩ, Sur une fonction transcendante et ses applications à la sommation de quelques séries, Ann. École Norm. Sup. (3) 21 (1904), 207-267, 459-533.

31. - Sur la développement, à l'aide des fonctions cylindriques, des sommes doubles $\sum f\left(p m^{2}+2 q m n+r n^{2}\right)$, où $p m^{2}+2 q m n+r n^{2}$ est une forme positive à coefficients entiers (verhandlungen des Dritten Internat. Math.-Kongr, Heidelberg), Teubner, Leipzig, 1905, pp. 241-245.

32. G. N. Watson, Theory of Bessel functions, 2nd ed., Cambridge Univ. Press, Cambridge, 1944. MR 6, 64.

33. J. R. Wilton, On Dirichlet's divisor problem, Proc. Roy. Soc. A 134 (1931), 192-202.

34. —_, Voronoi's summation formula, Quart. J. Math. Oxford Ser. 3 (1932), 26-32.

35. - An approximate functional equation with applications to a problem of Diophantine approximation, J. Reine Angew. Math. 169 (1933), 219-237.

UNIVERSITY OF ILLINOIS,

URBANA, ILLINOIS 61801 